\title{
Avoiding Regret in an Agent-Based Asset Pricing Model
}

\begin{abstract}
We use an agent-based asset pricing model to test the implications of the disposition effect (avoiding regret) on investors' interactions and price settings. We show that it has a direct impact on the returns series produced by the model, altering important stylized facts such as its heavy tails and volatility clustering. Moreover, we show that the horizon over which investors compute their wealth has no effect on the dynamics produced by the model.
\end{abstract}

Keywords: agent-based model, asset pricing, disposition effect, behavioural bias

\section{Introduction}

One of the building blocks of modern financial theory relies on the belief that asset prices are determined by the market interactions of utility maximising agents that form rational expectations about future outcomes. This is the theoretical underpinning of the efficient market hypothesis (Fama, 1970), which for a long time has been used to explain the behaviour of asset prices. In a setting where all agents are fully rational, they are assumed to follow the expected utility theory (von Neumann and Morgenstern, 1944) for making decisions under uncertainty.

However, this belief has been challenged over the years. Alternative theories describing how decision makers behave under uncertainty have been proposed, leading to the development of prospect theory (Kahneman and Tversky, 1979). Some of its important implications are that, in reality, people deviate from rationality and use biases or heuristics in decision making (Barberis and Thaler, 2003). Specifically, Shefrin and Statman (1985) use prospect theory to show that investors may hold losing trades longer than profitable ones - a now well-known phenomenon labelled as the disposition effect. Furthermore, evidence suggests that investors' unwillingness to cut their losses is closely related to the notion of regret, which may be accentuated by having to admit their mistakes to other people (Kahneman et al., 1982). Put differently, investors may not realise a loss because they are trying to avoid the regret associated with their initial (wrong) judgement.

Although behavioural biases have been clearly observed in real-life settings, examining their implications on markets is still a challenging topic. For this reason, a considerable focus has shifted towards developing models of human psychology as it relates to financial markets (Shiller, 2003). In particular, heterogeneous agent-based models that rely on simple trading strategies have proven themselves very efficient in generating important dynamics of real markets (Hommes, 2006). They attempt to explain these properties endogenously, by considering the interaction of market participants. Moreover, a well-defined agent-based model is an important tool for testing behavioural (Pruna et al., 2016) and economical theories (Chen, 2016) and understanding their influence on the interplay between agents and prices.

To this end, we extend the well-known structural stochastic volatility model of Franke and Westerhoff (2012) (FW) - one of the most successful in capturing empirically observed traders' behaviour (Barde et al., 2015). Specifically, we test, for the first time, the implications of the disposition effect on both agents' interactions and the asset price dynamics. We find that while the time horizon over which investors consider their wealth has no impact on the overall setting, the level of regret directly impacts the returns series produced by the model, altering its stylized facts and leading to disruptive behaviour.

\section{Model definition}

First, we describe the FW model, which we then extend to capture the disposition effect in agents' behaviour. Here, the asset price changes are determined by excess demand, following the interaction of two types of 
agents: fundamentalists and chartists. By excess demand we mean the precise positive or negative orders per trading period. Fundamentalists believe in the price reversal towards its perceived fundamental value and have a demand function, $d_{t}^{f}$, proportional to the gap $p_{t}^{f}-p_{t}$, where $p_{t}$ and $p_{t}^{f}$ are the asset's log price and fundamental log value at time $t$, respectively. We depart from the original FW setting and assume the fundamental price follows a geometric Brownian motion, as this modification was shown to overcome the stationary dynamics of price series generated by the model (Pruna et al., 2016). In contrast, chartists are simple trend followers whose demand is proportional to the latest price change. Thus, the net demand of each group for the asset in period $t$ is given by:

$$
\begin{array}{lll}
d_{t}^{f}=\phi\left(p_{t}^{f}-p_{t}\right)+\epsilon_{t}^{f}, & \epsilon_{t}^{f} \sim \mathcal{N}\left(0, \sigma_{f}^{2}\right), & \phi>0, \epsilon_{f}>0, \\
d_{t}^{c}=\chi\left(p_{t}-p_{t-1}\right)+\epsilon_{t}^{c}, & \epsilon_{t}^{c} \sim \mathcal{N}\left(0, \sigma_{c}^{2}\right), & \chi \geq 0, \epsilon_{c} \geq 0,
\end{array}
$$

where $\phi$ and $\chi$ are constants denoting the aggressiveness of the traders' demand and $\sigma_{t}^{f}$ and $\sigma_{t}^{c}$ are noise variances meant to capture the wide variety of within-group specifications.

The agents can switch between strategies at each iteration, with their market fractions $\left(n_{t}^{f}\right.$ and $\left.n_{t}^{c}\right)$ fluctuating over time. The total (scaled) demand given by $D_{t}=n_{t}^{f} d_{t}^{f}+n_{t}^{c} d_{t}^{c}$ will generally not add up to zero and we will have an excess of either supply or demand. A market maker is assumed to mediate the transactions between investors and provide liquidity. Specifically, the market maker reacts to the imbalance between demand and supply by proportionally adjusting the price with a constant factor $\mu>0$. Accordingly, the equation determining the price for the next period $t+1$ is given by:

$$
p_{t+1}=p_{t}+\frac{\mu}{2}\left(n_{t}^{f} d_{t}^{f}+n_{t}^{c} d_{t}^{c}\right)+\epsilon_{t}, \quad \epsilon_{t} \sim \mathcal{N}\left(0, \sigma_{t}^{2}\right)
$$

where the combined time-varying variance $\sigma_{t}$ depends on the changes in the market fractions of the fundamentalists and chartists.

For completeness, it is necessary to set up the motions of market fractions, determined by a transition probabilities approach (Lux, 1995). Its basic idea is that agents switch strategies with certain probabilities, relying on the exponential of a switching index, $a_{t}$, such that:

$$
\begin{gathered}
P_{t}^{c f}=\nu \exp \left(a_{t}\right) \\
P_{t}^{f c}=\nu \exp \left(-a_{t}\right),
\end{gathered}
$$

where $P_{t}^{c f}, P_{t}^{f c}$ are the probabilities of shifting from chartist to fundamentalist at time $t$ and vice-versa, respectively. The parameter $a_{t}$ is determined by several factors including a predisposition towards one of the two types, a herding factor and a price misalignment between $p_{t}$ and $p_{t}^{f}$. These three elements combined form the attractiveness level, formally defined as:

$$
a_{t}=\alpha_{0}+\alpha_{n}\left(n_{t}^{f}-n_{t}^{c}\right)+\alpha_{p}\left(p_{t}-p_{t}^{f}\right)^{2},
$$

where $\alpha_{0}$ is the predisposition parameter, $\alpha_{n}$ captures the herding parameter and $\alpha_{p}$ measures the influence of price misalignment.

Now, since the FW model has been shown to recreate an extensive list of statistical properties of real-life financial markets, we choose it to test the implications of investors' unwillingness to cut their losses.

In their seminal paper, Kahneman et al. (1982) argue that people think in terms of changes in their wealth, being concerned about its fluctuations. After a loss in the stock market, an investor may experience a sense of regret over his decision of using a particular strategy (Wong, 2015). He may interpret his loss as a sign that his thinking is flawed and he may feel disappointment if word leaks out (Barberis et al., 2001). Therefore, to avoid the regret associated with his judgement being wrong, he has a disposition to stick with his strategy for a longer period of time.

To incorporate this behavioural bias in our agent-based model, we need to formally define the notion of a loss. In more detail, with respect to strategy $s=f, c$, let $g_{t}^{s}$ be the short term capital gains that an agent of this type realises at time $t$ :

$$
g_{t}^{s}=\left[\exp \left(p_{t}\right)-\exp \left(p_{t-1}\right)\right] d_{t-2}^{s},
$$


and let

$$
w_{t}^{s}=\eta w_{t-1}^{s}+(1-\eta) g_{t}^{s},
$$

be the profit earned by an agent that follows strategy $s$, discounted by a memory coefficient $\eta<1$.

Some of the agents are assumed to be biased towards retaining losing positions in order to avoid the regret of being wrong ${ }^{1}$. As a result, whenever they find themselves in a losing strategy they do not realise the loss and stick to that strategy hoping that their bad luck will soon reverse. Formally, the probability of the biased agents switching types decreases when they are losing money:

$$
\begin{array}{ll}
\hat{P_{t}^{c f}}=P_{t}^{c f}-\lambda P_{t}^{c f}, & \text { if } w_{t}^{c}<0, \\
\hat{P_{t}^{f c}}=P_{t}^{f c}-\lambda P_{t}^{f c}, & \text { if } w_{t}^{f}<0,
\end{array}
$$

where $\lambda \in[0,1]$ measures the agents' unwillingness to cut their losses, avoiding regret. We can view $\lambda$ as the percentage of biased investors within the population.

A further important concept in prospect theory is the horizon over which gains and losses are measured - in other words, how often an agent evaluates his investment performance. In our model, this is specified by the memory parameter $\eta \in[0,1)$, which indicates how much weight an agent puts on his latest profits and how much on his past wealth, when computing his current, accumulated wealth. Simply put, $\eta$ relates to the look-back window used by an agent when calculating his wealth, with the time horizon increasing with $\eta$. Since the disposition effect is associated with the investors' tendency of ignoring past mistakes and putting them in a larger perspective, the memory parameter should play a role in how this bias affects the agents.

Note that the attractiveness level and the two parameters $\eta$ and $\lambda$ are all independent of each other. In the original $\mathrm{FW}$ setting, the market fractions are determined by the transition probability approach, depending only on $a_{t}$. However, in order to capture the agents' unwillingness to cut their losses, our extended version of the model introduces two new parameters, $\eta$ and $\lambda$. Furthermore, since our aim is to test the implications of the behavioural bias on agents' interactions and returns settings, we will mainly focus on these two parameters.

Clearly, both $\eta$ and $\lambda$ have an impact on the market interactions, independent of $a_{t}$. On one hand, $\eta$ puts into larger perspective the latest realized loss when calculating the current level of wealth. The larger the realised loss $g_{t}^{s}$, the larger its impact on the accumulated wealth when multiplied by $1-\eta$ and thus the less likelihood of switching strategies. On the other hand, the impact of $\lambda$ directly follows from Equation 8 . As $\lambda$ increases, agents become biased and the likelihood of switching strategies decreases. Finally, for large values of $\lambda$, as the percentage of biased agents increases, it dominates the switching mechanism.

\section{Impact of the disposition effect}

To assess the implications of investors' unwillingness to realise their losses, we vary both the regret $\lambda \in[0,1]$ and the memory $\eta \in[0,1)$ parameters, considering 100 equally spaced values for each parameter. We fix all the other parameters to their values reported by Franke and Westerhoff (2012), where they were estimated using the method of simulated moments on observed S\&P500 data. Interestingly, Figures 1 (a) and (b) shows that the agents' market fractions are not notably affected by either $\lambda$ or $\eta$, having a median value of $n_{f} \approx 0.93$. This is due to the stochastic behaviour of prices leading to the wealth changing from positive to negative values. Therefore, the changes in the transition probabilities balance out and market proportions don't change much, with both fundamentalist and chartists surviving the competition, as we usually see in these settings.

We now turn our attention to the implications of memory and regret on the returns series produced by our behavioural model. The returns themselves are specified in percentage points, so with respect to the

\footnotetext{
${ }^{1}$ Here we investigate only one aspect of the disposition effect, namely, holding onto the losing position. The other tendency of selling the winners too soon is not incorporated in our model.
} 


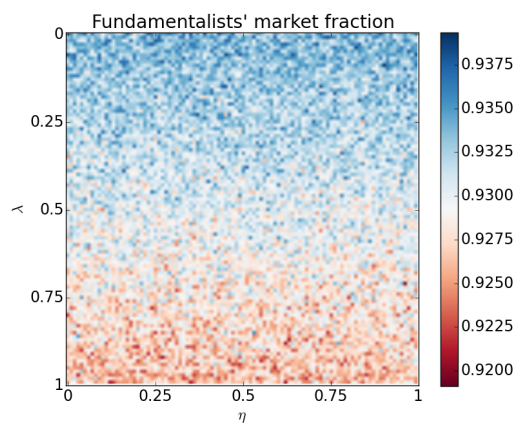

(a)

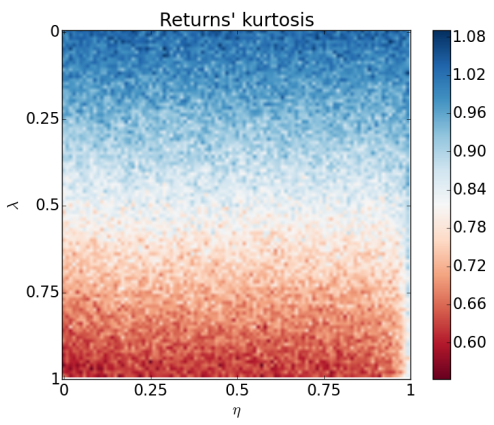

(c)

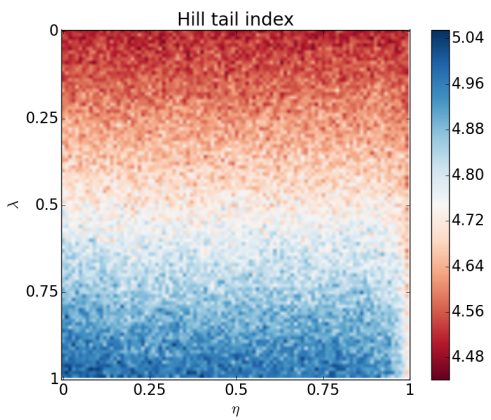

(e)

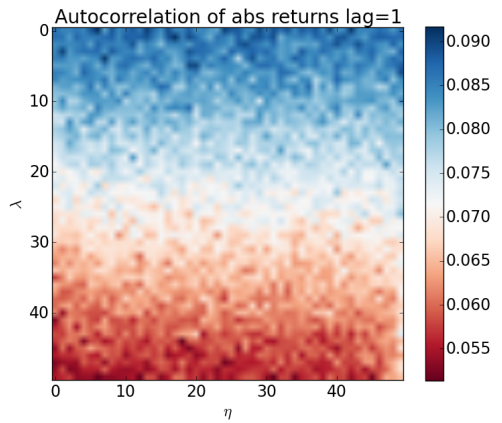

(g)

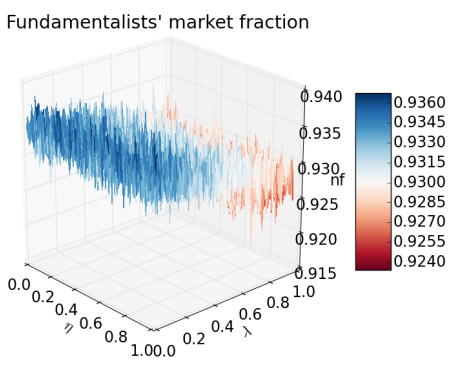

(b)



(d)

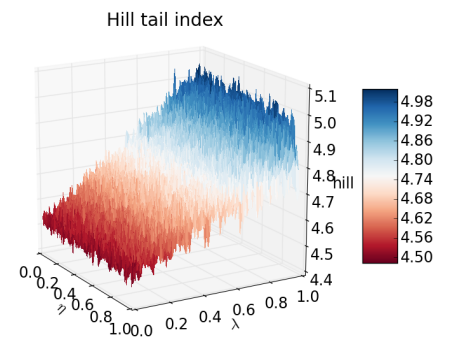

(f)

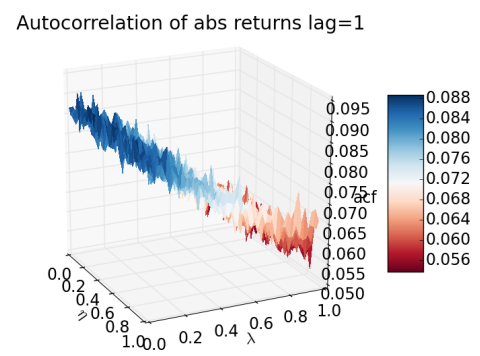

(h)

Figure 1: Impact of the disposition effect on agents' interactions and returns. 
$\log$ prices $p_{t}$, we may write $r_{t}=100 *\left(p_{t}-p_{t-1}\right)$. In Figures 1 (c), (d), (e), (f), (g) and (h) we plot the returns' excess kurtosis, its Hill tail index and the autocorrelation of absolute returns for different levels of $\lambda$ and $\eta$. We observe that the memory parameter doesn't affect the returns at all. This leads to the counter intuitive finding that the horizon over which wealth is measured doesn't change the agents' interactions or the model's dynamics. Therefore, the investors' unwillingness to cut their losses has the same effect on the overall dynamics irrespective of how often they evaluate their performance.

On the other hand, the regret parameter has a direct impact on the properties of returns. As $\lambda$ increases, its excess kurtosis drops 40\% from 1.00 to 0.60, while the Hill tail index grows $11 \%$ from 4.50 to 5.00. This means the peakiness of returns and its tail heaviness decrease as the agents become more biased, leading to a flatter and more normal distribution of returns. In other words, we observe a decrease in the heavy tails of returns. Moreover, Figures $1(\mathrm{~g})$ and $(\mathrm{h})$ show how the first autocorrelation of absolute returns decreases $42 \%$ from 0.93 to 0.54 as we increase $\lambda$. This is a clear sign of decline in volatility clustering.

Therefore, an increase in the disposition effect leads to violations of some of the most discussed stylized facts of financial data ${ }^{2}$. Note that the outbursts in volatility appear as a consequence of chartism dominance periods. Accordingly, in a setting where $p_{t}$ deviates from $p_{t}^{f}$, the fundamentalists lose money and their bias lowers the probability of shifting to chartism. Moreover, if the price follows the trend, the chartists make profit and their probability of switching to fundamentalism is not affected by the disposition effect. Accordingly, the fundamentalists' market fraction stays high, with less outburst of chartism and volatility clustering. Overall, investors unwillingness to cut their losses has a significant impact on the time series produced by our model.

\section{Conclusions}

Our work leads to two important findings. On the one hand, we show that the horizon over which the agents consider alternative scenarios, an important concept of prospect theory, has no impact on the setting dynamics. This is surprising because in prospect theory it does have a decisive role in agents' interactions.

On the other hand, the level of regret directly impacts the returns series produced by the model, altering its stylized facts and leading to disruptive behaviour. Specifically, we demonstrate how these disruptions can appear and show that the disposition effect has a direct impact on the returns' distribution, its heavy tails and volatility clustering. This provides support for the behavioural finance literature arguing that investors systematic biases can lead to unexpected market behaviour, with deviations from efficiency and rationality.

For future work, we will use the model to investigate the impact of the other side of the disposition effect (selling winners too soon) and other behavioural factors such as gossip or waves of optimism and pessimism on the system's dynamics.

Barberis, N., Huang, M., Santos, T., 2001. Prospect theory and asset prices. The Quarterly Journal of Economics 116, 1-53. Barberis, N., Thaler, R., 2003. A survey of behavioral finance. Handbook of the Economics of Finance 1, 1053-1128.

Barde, S., et al., 2015. Direct calibration and comparison of agent-based herding models of financial markets. Technical Report. School of Economics, University of Kent.

Chen, H., 2016. A tobin tax only on sellers. Finance Research Letters 19, 83-89.

Fama, E.F., 1970. Efficient capital markets: A review of theory and empirical work. The Journal of Finance 25, 383-417.

Franke, R., Westerhoff, F., 2012. Structural stochastic volatility in asset pricing dynamics: Estimation and model contest. Journal of Economic Dynamics and Control 36, 1193-1211.

Hommes, C.H., 2006. Heterogeneous agent models in economics and finance. Handbook of Computational Economics 2, $1109-1186$.

Kahneman, D., Tversky, A., 1979. Prospect theory: An analysis of decision under risk. Econometrica: Journal of the Econometric Society, 263-291.

Kahneman, D., Tversky, A., et al., 1982. The psychology of preferences .

Lux, T., 1995. Herd behaviour, bubbles and crashes. The Economic Journal , 881-896.

\footnotetext{
${ }^{2}$ For values of $\eta$ close to $1(\eta>0.995)$, there are no disruptions in the returns' stylized facts. For such large values of $\eta$, the impact of the latest realized profit or loss will be very small on the total wealth. Since the price misalignment component ensures that the market price doesn't departs too much from the fundamental one, the agents' wealth would stay positive over time. This means that the behavioural bias won't be activated and the new model won't differ from the original one
} 
von Neumann, J., Morgenstern, O., 1944. Theory of games and economic behavior.

Pruna, R.T., Polukarov, M., Jennings, N.R., 2016. An asset pricing model with loss aversion and its stylized facts. Computational Intelligence for Financial Engineering \& Economics (CIFEr), IEEE Symposium Series on , 1-8.

Shefrin, H., Statman, M., 1985. The disposition to sell winners too early and ride losers too long: Theory and evidence. The Journal of Finance 40, 777-790.

Shiller, R.J., 2003. From efficient markets theory to behavioral finance. The Journal of Economic Perspectives $17,83-104$.

Wong, K.P., 2015. A regret theory of capital structure. Finance Research Letters 12, 48-57. 